\title{
Improved service through renal transplant consent clinic despite low proportion of patients requiring additional investigations
}

Rachael Czajka,Vivek Upasani, Richard Baker, Clare Ecuyer, Natalie Reeves, Kate Brady, Heather Roberts, Vijayanand Dhakshina, Niaz Ahmad, Lutz Hostert, Adam Barlow, Matthew Welberry Smith, St James' University Hospital, Leeds.

\section{Introduction}

British Transplant Society (BTS) guidelines state that for patients on the Renal Transplant waiting list, "consent should be re-affirmed at least every 12 months where possible; this should be done by an appropriately experienced and trained health care professional."'

Renal transplants have been carried out at St James' University Hospital, Leeds since 1967. Since that time over 3,000 transplants have taken place. We provide a service for patients throughout East, West and North Yorkshire, and the Humber. At the time of data collection, there were 407 patients on the regional waiting list; 217 patients active and 380 suspended.

A dedicated Consent Clinic was formed in Leeds in May 2015, in keeping with BTS guidelines. At one year, we have reviewed the data relating to this clinic, including attendance rates, additional tests ordered, and changes to patient waiting list status.

\section{Methods}

Quantitative analysis of all clinic appointments in the first year of clinic: number of patients seen, cancellation and DNA rates, tests ordered, and changes made to transplant status. Data was obtained using clinic letters, results server and BHLY, the regional Renal database for Bradford, Hull, Leeds and York.

\section{Results}

A total of 447 appointments were sent in the first year. There were 255 attendances, I 50 cancellations and 42 DNAs. Forty four patients came to a subsequent appointment after cancelling or not attending. There was no difference in cancellation / DNA rates for geographically distant patients from more remote referring units vs. more central units.

229 patients were seen by a Surgeon, 5 seen by a Renal Physician and 20 seen by both. Thirty eight patients (I5\%) had investigations requested, including $27 \mathrm{MRI}$ scans and 23 cardio-respiratory investigations (see Figure 2). Two patients were referred to other specialties (Vascular and Cardiology) and one was worked up for a transplant nephrectomy.

Thirteen patients $(5 \%)$ in total were suspended from waiting list based on clinic review. Reasons for suspension included high BMI, having a concurrent illness, and awaiting test results. Two patients were permanently removed from the waiting list - one due to a high risk CPEX and the other due to extensive vascular disease.

Figure I. Changes to Waiting List Status

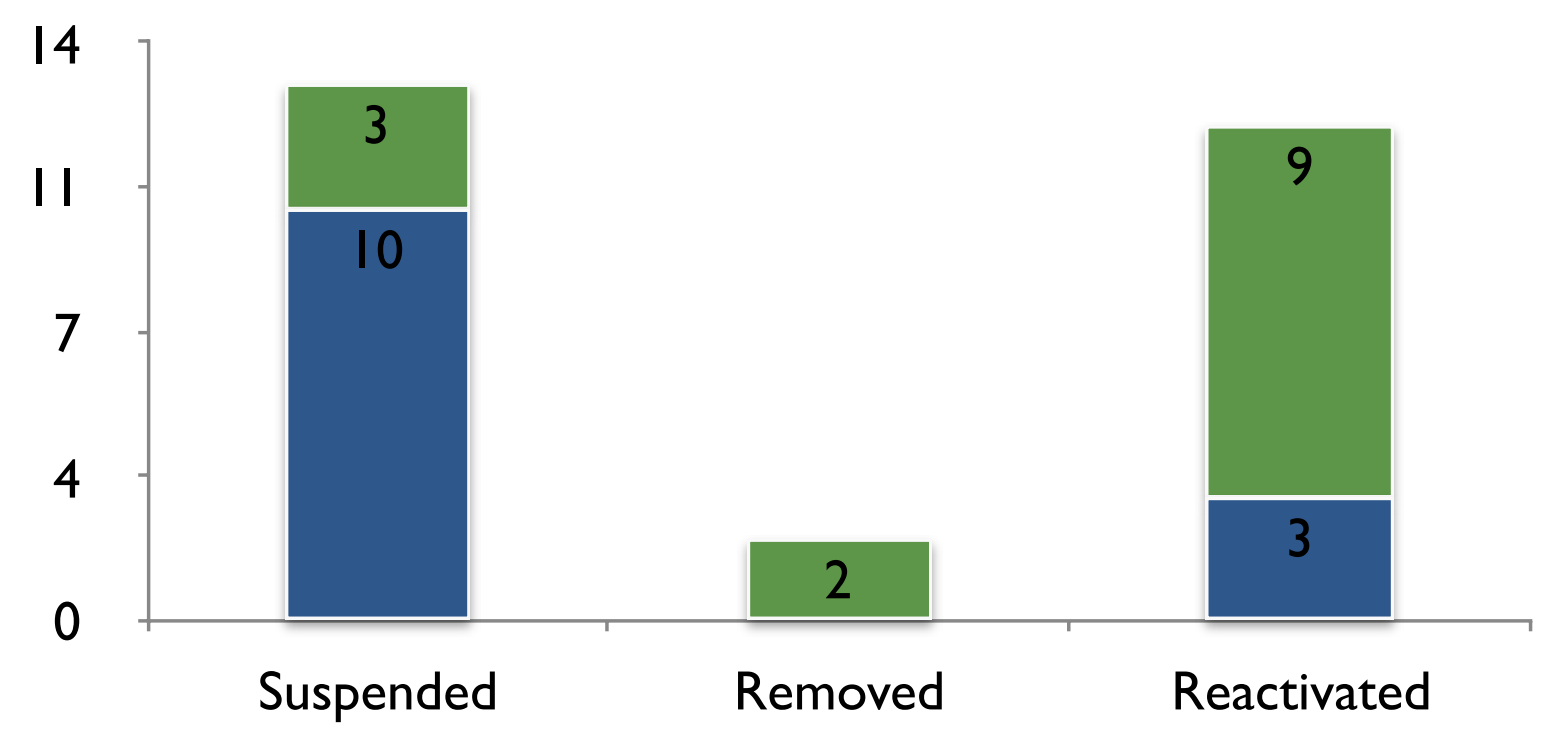

Initial Changes Changes With Results
Initial patient feedback has been extremely positive, commenting on the chance to meet the transplant team and ask questions. Patients described the experience as a 'dummy run' which allowed them to familiarise themselves with the hospital, and the team.

Transplant co-ordinator feedback highlighted the opportunity to check contact details, reiterate the importance of contactability and remind patients to inform staff of any sensitising events. It also gave an opportunity to discuss live related donation.

Surgeon feedback raised the benefits of assessing general fitness and the need for further investigations, as well as assessing suitability for en bloc and dual transplant.

Figure 2. Percentages of Investigations Requested (out of 56 ordered in total)

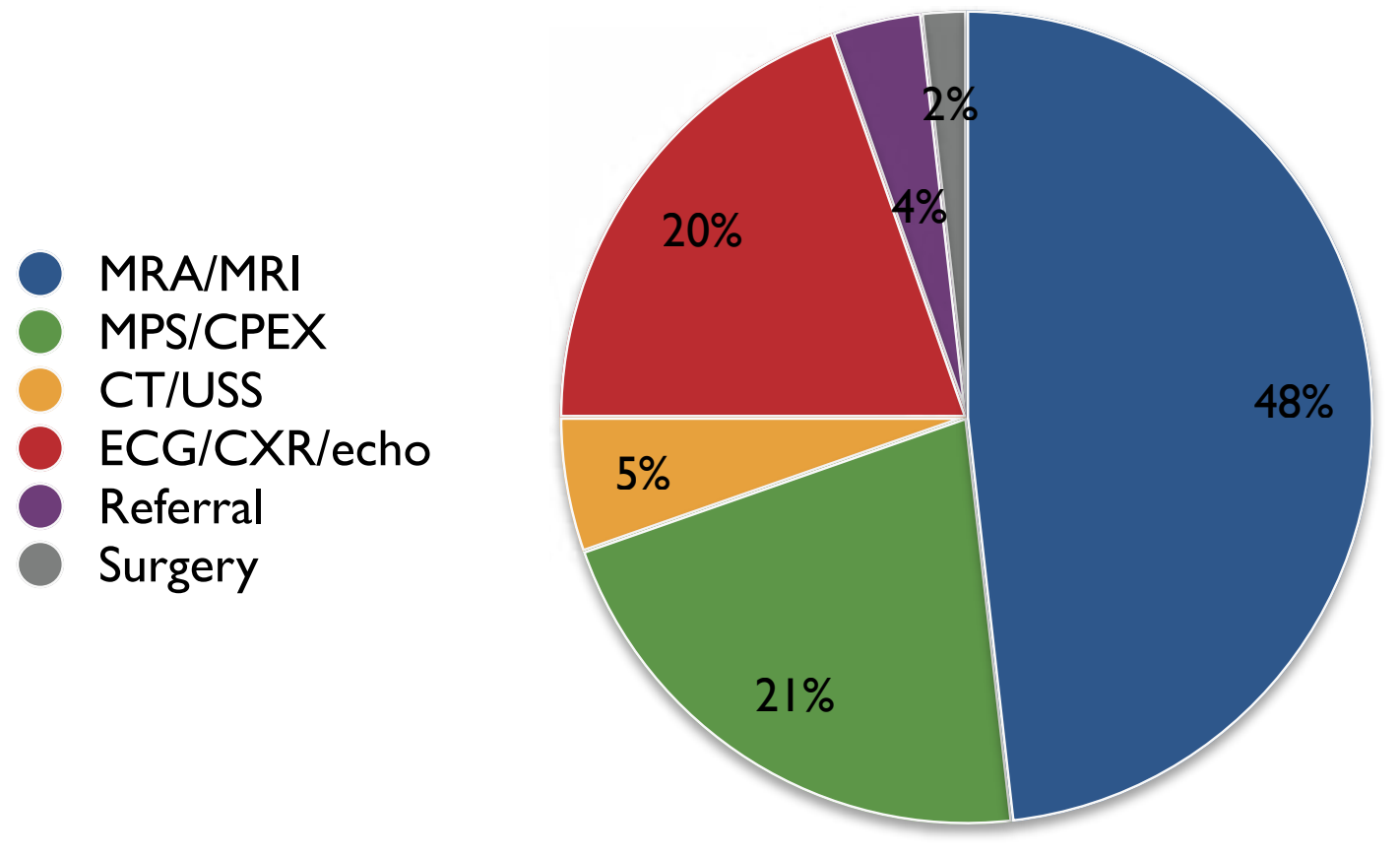

\section{Discussion}

Although there were low numbers of changes to transplant list status, having a dedicated consent clinic provided an improved service, and ensured compliance with BTS guidelines.

In addition to providing a consent service, the clinic also offers an opportunity to identify patients who require more up to date investigations, or who may have developed co-morbidities which could inhibit fitness for transplant.

The transplant team have increased confidence that patients who are called in for transplant will be fit to proceed, and there have been no instances of patients being deemed unsuitable at the time of presentation since the Consent Clinic began. Patients valued the opportunity to meet the team and see the transplant facilities. In addition to positive feedback, formal patient outcome measures analysis will be performed as a next step, as well as a review of the effect of introducing anaesthetic input to clinics. 